\title{
Physical models of streaming instabilities in protoplanetary discs
}

\author{
Jonathan Squire ${ }^{\circledR 1 \star}$ and Philip F. Hopkins ${ }^{\circledR 2}$ \\ ${ }^{1}$ Physics Department, University of Otago, Dunedin 9016, New Zealand \\ ${ }^{2}$ TAPIR 350-17, California Institute of Technology, Pasadena, CA 91125, USA
}

Accepted 2020 July 31. Received 2020 July 29; in original form 2020 February 29

\begin{abstract}
We develop simple, physically motivated models for drag-induced dust-gas streaming instabilities, which are thought to be crucial for clumping grains to form planetesimals in protoplanetary discs. The models explain, based on the physics of gaseous epicyclic motion and dust-gas drag forces, the most important features of the streaming instability and its simple generalization, the disc settling instability. Some of the key properties explained by our models include the sudden change in the growth rate of the streaming instability when the dust-to-gas mass ratio surpasses one, the slow growth rate of the streaming instability compared to the settling instability for smaller grains, and the main physical processes underlying the growth of the most unstable modes in different regimes. As well as providing helpful simplified pictures for understanding the operation of an interesting and fundamental astrophysical fluid instability, our models may prove useful for analysing simulations and developing non-linear theories of planetesimal growth in discs.
\end{abstract}

Key words: instabilities - planets and satellites: formation - protoplanetary discs.

\section{INTRODUCTION}

Understanding the mechanisms that enable planetesimal formation within protoplanetary discs continues to represent an outstanding challenge in theoretical astrophysics (Chiang \& Youdin 2010). An initial population of micron-sized dust grains entrained in the disc must coagulate and grow many orders of magnitude in size in order to collapse gravitationally. They must do so remarkably quickly, given the rapid infall of moderate-sized bodies (Goldreich \& Ward 1973; Nakagawa, Sekiya \& Hayashi 1986) and the short lifetimes of observed discs (see e.g. Ansdell et al. 2017). A promising solution involves the clumping of grains through collective fluid-dynamical instabilities driven by the relative motion of dust through the gas (Goodman \& Pindor 2000). The so-called 'streaming instability,' discovered by Youdin \& Goodman (2005), is seen in non-linear simulations to cause significant clumping of dust, allowing gravitational collapse into planetesimals under the right conditions (see e.g. Johansen et al. 2007, 2014; Simon et al. 2016; and references therein). While there remain some potential issues - in particular, the supersolar metallicities and relatively large grain sizes that seem to be needed for robust planetesimal formation (e.g. Johansen, Youdin \& Mac Low 2009; Bai \& Stone 2010; Yang, Johansen \& Carrera 2017) - the general mechanism seems robust, particularly if accompanied by other grain concentration mechanisms such as other collective dust-gas instabilities (Squire \& Hopkins 2018b), gas evaporation (Williams \& Cieza 2011), or gas inhomogeneities (Birnstiel, Fang \& Johansen 2016 and references therein).

However, despite its likely astrophysical importance, the streaming instability lacks any simple, intuitive model for its operation, a situation that is somewhat unique among the broad landscape of

^E-mail: jonathan.squire@otago.ac.nz astrophysical fluid and plasma instabilities (e.g. the magnetorotational instability; Balbus \& Hawley 1992). In this article, we work to provide such models to explain the linear growth of dustgas streaming instabilities, building on Squire \& Hopkins (2018b) (hereafter Paper I) and a variety of previous studies by other authors (Youdin \& Goodman 2005; Youdin \& Johansen 2007; Jacquet, Balbus \& Latter 2011; Auffinger \& Laibe 2018; Jaupart \& Laibe 2020). Our models explain all the key features of the instabilities, including their dependence on physical parameters, their linear mode structure, and their growth rates. As well as the traditional streaming instability of Youdin \& Goodman (2005), we also consider the related 'settling instability' of Paper I, which relies on very similar physics and may clump small grains more efficiently than the streaming instability as they settle into the disc mid-plane.

Our primary motivation for this work is to improve the general theoretical understanding of a commonly studied mechanism that appears to be crucial to the development of planetary systems. For example, our models explain, based on simple physical principles, the sudden change in the growth rate of the streaming instability as the dust-mass fraction surpasses one, and the striking difference in growth rates between the streaming and settling instabilities. In addition, such models may be able play a more practical role in future work by, for example, helping to diagnose and understand grain clumping in more realistic non-linear simulations. They may also be useful for developing non-linear models of streaminginstability-generated turbulence, in a similar way to a variety of other astrophysical fluid instabilities, for example, convection (e.g. Collins 1989), plasma instabilities (e.g. Melrose 1980), or related dust instabilities (Moseley, Squire \& Hopkins 2019; Seligman, Hopkins \& Squire 2019; Hopkins, Squire \& Seligman 2020).

Before discussing the streaming instability in detail, we feel it is helpful to provide a single, simplified sketch of its mechanism in Fig. 1. This illustrates how the basic physical ingredients of drag 


\section{Mechanism}

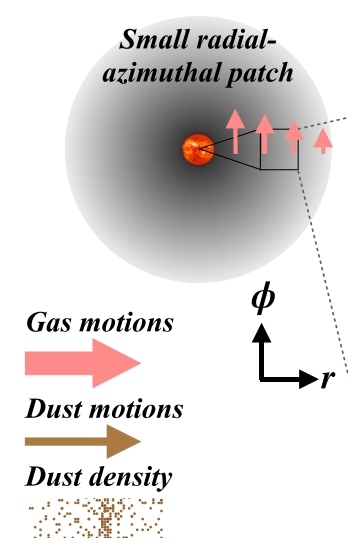

(1) Dust clump drifts radially inwards

(2) Dust backreaction drags gas inwards around the dust clump

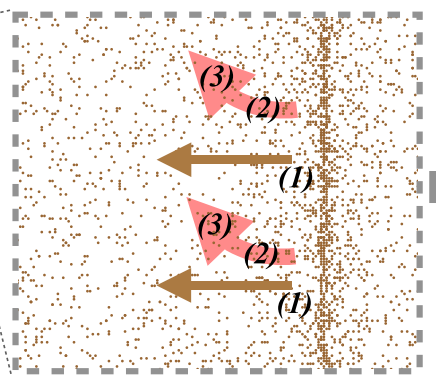

(3) Inwards flow deflected into azimuthal flow by Coriolis force
(4) Gas drags dust, causing superKeplerian dust velocity

(5) Super-Keplerian flow of dust causes outwards deflection compared to background drift

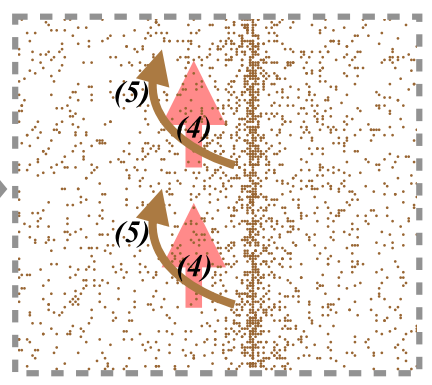

(6) Dust piles up, enhancing the dust density in the clump

\section{Constraint}

If perturbation is purely radial, radial gas motions (steps 2-3) are halted by strong thermal pressure forces

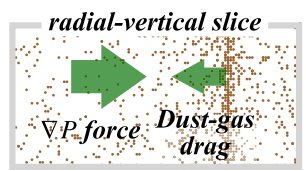

This kills the feedback mechanism

Vertical gas motions allow for incompressible radial gas motions

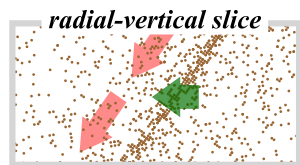

Figure 1. Summary figure showing the key physical processes that enable the streaming instability. Steps (1)-(6) in 'Mechanism' illustrate the azimuthal-radial plane to show how axisymmetric dust and gas motions can enhance a dust density to maximum as it drifts inwards due to a combination of drag forces ( 1,2 , and 4) and Coriolis and shear forces ( 3 and 5). However, such motions are only possible if perturbations vary vertically, as well as radially, as illustrated in 'Constraint.' The sketched mechanism is overly simplified in order to capture the key physical principles; Figs 4,5 , and 7 give a more accurate representation of the feedback loops that drive the instability across its different regimes.

and Coriolis forces can conspire to enhance a drifting dust-density clump, so long as the clump also has vertical structure. We believe that this is the simplest reasonable representation of the physics of the streaming instability (although it remains overly simplified), and may satisfy the reader who desires a basic understanding of its key features. The physics driving various unstable modes in different regimes is thoroughly described throughout the rest of this paper.

\subsection{The philosophy of this article}

Given our desire to formulate simple, physical explanations for dust clumping in discs, the approach of this article is to avoid, wherever possible, detailed mathematical presentation of growth rates and mode structure. Rather, we attempt to distill each case down to the simplest possible explanation that correctly captures its physical characteristics. Our models are, thus, deliberately rather qualitative. Nonetheless, we wish to emphasize that each was derived using the linearized equations of motion for the coupled dustgas system, employing understanding gained using the 'Resonant Drag Instability' (RDI) theory of Squire \& Hopkins (2018a). As an example, the response of the dust density to an epicycle, which will be introduced in Section 3.2 and used extensively throughout the article, can be derived through the direct solution of the four linearized dust equations, forced by a periodic gas velocity. We invite the reader interested in further mathematical details to consult Paper I. A related mathematical discussion, which focuses primarily on the settling instability, is found in Zhuravlev (2019), and the recent papers of Pan \& Yu (2020) and Pan (2020) discuss a variety of similar concepts from a somewhat more mathematical perspective.

With the same spirit, throughout the article, we will neglect all non-ideal physical effects that would complicate the discussion; we consider only a single population of grain sizes and neglect the effects of background gas turbulence, gas viscosity, and dust diffusion or dispersion. Such effects can be very important and their neglect here is motivated only by our desire to keep the models as simple as possible. Indeed, a distribution of grains has been shown to strongly impede the growth of the streaming instability in the low-dust-to-gasratio regime (Krapp et al. 2019), while turbulence and/or viscosity can significantly limit the applicability of the instability in many scenarios (e.g. Squire \& Hopkins 2018b; Umurhan, Estrada \& Cuzzi 2019; Chen \& Lin 2020; Jaupart \& Laibe 2020; Krapp et al. 2020; Zhuravlev 2020). Our models could, nonetheless, prove useful as the basis for more complex models of instabilities in systems with distributions of grain sizes, while turbulence, viscosity, or dust diffusion could likely be straightforwardly added, if desired (for example, viscosity will impede the growth of modes below some scale).

Following a brief description of the local approximation, dust drifts (Section 2.1), and the physics of coupled dust-gas dynamics (Section 2.2), our presentation starts with an analysis of how dust responds to gas epicycles (Section 3.2), and how the gas responds to dust-density perturbations (Section 3.3). Coupled to the idea of resonance between drifting dust and gas modes (the basis for RDI theory), these responses explain the characteristics of the lowdust-to-gas-ratio streaming instability in Section 4 and the settling instability in Section 5, clarifying why they exhibit vastly different growth rates for small grains. As noted in Youdin \& Goodman (2005) and Paper I, the character of the streaming instability changes substantially when the dust-mass fraction is larger than unity and this regime must be considered separately. We explain this transition and the characteristics of the high-dust-to-gas-ratio streaming instability in Section 6. Our model explains its large growth rate, which is likely fundamental to the planetesimal formation process. Our models for each case are sketched in Figs 4, 5, and 7, which are intended to be understandable without detailed reference to the text.

\section{DUST-GAS INTERACTION IN DISCS}

\subsection{Conventions and definitions}

The instabilities that concern us in this article are all local in character. This means that rather than having to describe specific models for global density, temperature, and metallicity variations in a disc, we can consider just a local patch of near-Keplerian disc with constant background gas and dust densities and a constant linear shear profile. Of course, we assume that all instabilities studied vary on small scales compared to the global disc variation and if this is found a posteriori 
Table 1. Important symbols and definitions used throughout this article. The right-hand column notes useful considerations relating to the symbol in question and/or shows how symbols are illustrated graphically in Figs 2-7. The key background parameters that control instability properties are $\mu$ and $\tau_{s}$.

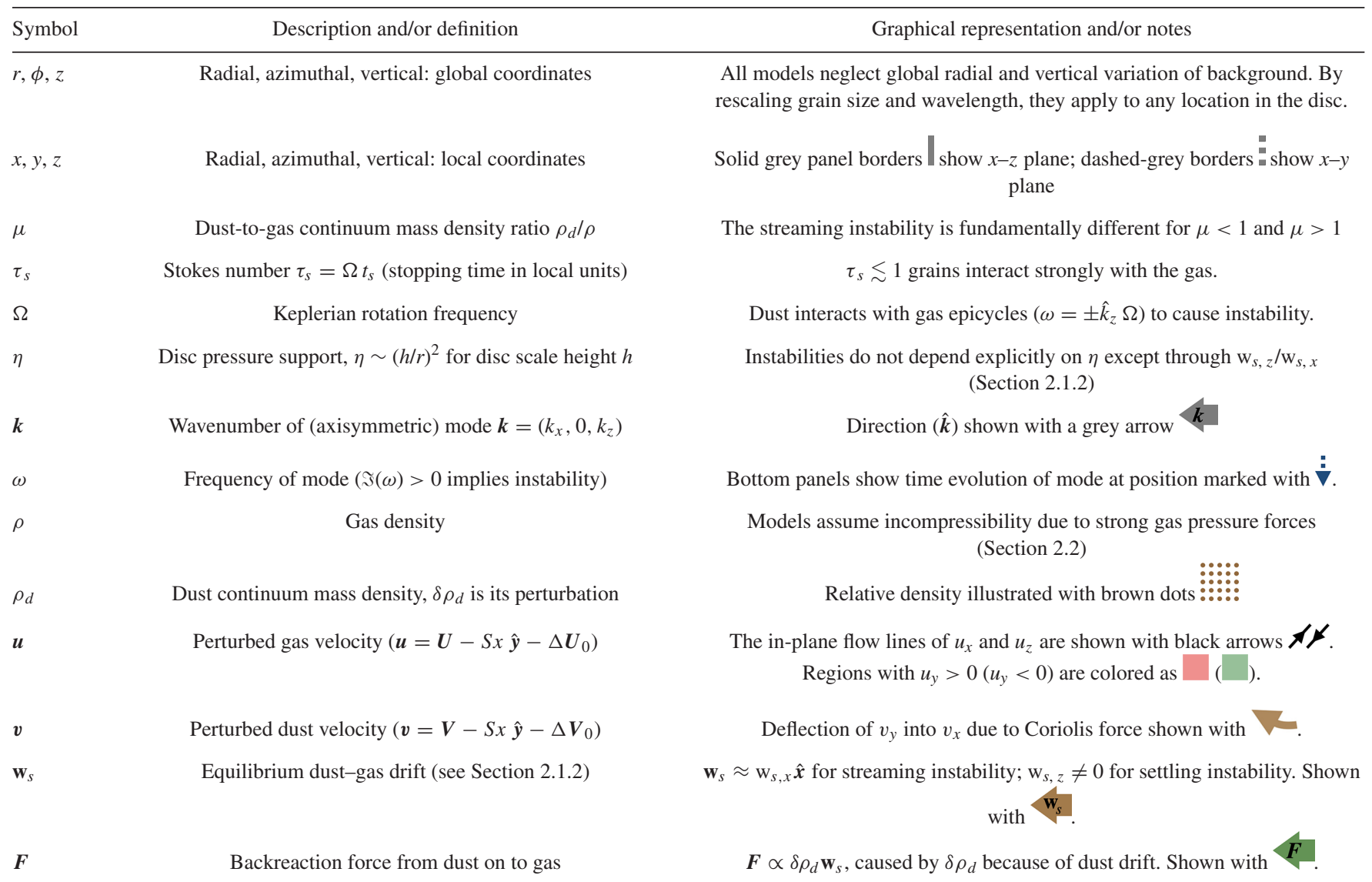

to not be true, our analysis is not valid. The great advantage of the local approach is simplicity, in particular, that our system depends only on two parameters: the dust size and the dust-to-gas mass ratio. We further assume that perturbations to the local equilibrium are very small, allowing one to derive linear equations for the spatiotemporal evolution of any quantity (velocity, density, etc.). Solutions to these equations can then be completely characterized by assuming that the perturbation of each fluid quantity follows the spatiotemporal variation $f_{\text {spat }}(\boldsymbol{x}, t)=f(\boldsymbol{k}, \omega) \exp (i \boldsymbol{k} \cdot \boldsymbol{x}-i \omega t)+$ conj., where $f_{\text {spat }}(\boldsymbol{x}, t)$ is a fluid perturbation (e.g. perturbed gas velocity or the variation in dust density), and $f(\boldsymbol{k}, \omega)$ is its complex Fourier amplitude. Due to linearity, the Fourier amplitudes drop out and $\omega$ satisfies a simple polynomial equation (the dispersion relation) with the wavevector $\boldsymbol{k}$ (magnitude $k$ and direction $\hat{\boldsymbol{k}}$ ) as a parameter. Further, since we are free to study the stability of any chosen mode separately from all other modes, the spatial variation of all quantities in our models is naturally sinusoidal. In keeping with convention, we use the directions $\hat{\boldsymbol{x}}, \hat{\boldsymbol{y}}$, and $\hat{z}$ to refer to the radial, azimuthal, and disc-normal (vertical) directions in the local frame, while $(r, \phi, z)$ refer to the global coordinate system. A number of useful definitions, including illustrations of the symbols used in later figures, are given in Table 1.

\subsubsection{Dust properties}

We assume the dust to be a pressureless fluid with average continuum mass density $\rho_{d}=\mu \rho$, where $\rho$ is the gas density and $\mu$ is the dustto-gas ratio. The dust interacts with the gas through drag forces governed by the stopping time $t_{s}$, which is the approximate time it takes a particle to come to rest in the frame of the gas. As in previous works, it is helpful to parametrize the dust size with the Stokes number, $\tau_{s} \equiv t_{s} \Omega$, where $\Omega$ is the Keplerian frequency in our local patch of disc. Particles with $\tau_{s} \lesssim 1$ can be considered 'strongly coupled' to the gas (drag forces are larger than rotational forces) and are the focus of this article. Understanding the coagulation of those in the range $10^{-4} \lesssim \tau_{s} \lesssim 1$, with physical sizes from the submillimeter scale up to somewhat less than a meter depending on the location in the disc, presents a number of interesting challenges to planet formation theory (Chiang \& Youdin 2010; Carrera, Johansen \& Davies 2015; Yang et al. 2017). It transpires that the detailed functional form of the dependence of $\tau_{s}$ on ambient gas properties - for example, whether the grains obey the Epstein or Stokes drag law - is not important for the discussion here, and our results can apply equally well at any physical location in the disc, so long as the dust size is scaled appropriately (the same $\tau_{s}$ corresponds to smaller particles further out in the disc). In formulating our models, we will sometimes assume $\tau_{s} \ll 1$, which is useful for understanding the general motions and forces on the dust and gas. However, the basic mechanisms we discuss for the streaming and settling instabilities are qualitatively applicable for all grains with $\tau_{s} \lesssim 1$.

\subsubsection{Pressure gradient and dust drifts}

The fundamental driver of the streaming and settling instabilities is the presence of global gas pressure gradients $\partial P / \partial \ln r$ and $\partial P / \partial \ln z$. 
The radial gradient $(\partial P / \partial \ln r)$ causes the gas motions to differ slightly (by $\Delta \boldsymbol{U}_{0}$ ) from circular Keplerian orbits (velocity $U_{K}$ ), with the proportional difference in azimuthal velocity $\left(\Delta U_{0, y} / U_{K}\right)$,

$\eta \equiv-\frac{\Delta U_{0, y}}{U_{K}} \approx-\frac{\partial P / \partial \ln r}{2 \rho U_{K}^{2}} \sim \frac{c_{s}^{2}}{U_{K}^{2}} \sim \frac{h^{2}}{r^{2}}$,

where $c_{s}$ is the gas sound speed and $h / r$ is the disc aspect ratio. Equation (1) has assumed a thin disc in vertical equilibrium, with pressure support dominated by thermal forces (weak turbulence and magnetic fields). The pressure-support-induced velocity difference $\Delta \boldsymbol{U}_{0}$, coupled to the gas-dust drag forces, induces an equilibrium velocity of the dust compared to Keplerian orbits, denoted $\Delta \boldsymbol{V}_{0}$. Because $\Delta \boldsymbol{V}_{0} \neq \Delta \boldsymbol{U}_{0}$, this causes a bulk drift of the dust in the frame of the gas, $\mathbf{w}_{s} \equiv \Delta \boldsymbol{V}_{0}-\Delta \boldsymbol{U}_{0}$, which provides the free-energy source for instability. In the mid-plane of the disc, where $\partial P / \partial \ln z=$ 0 , this is (Nakagawa et al. 1986)

$$
\frac{\mathbf{w}_{s}}{\eta U_{K} r} \approx-2 \frac{(1+\mu) \tau_{s}}{(1+\mu)^{2}+\tau_{s}^{2}} \hat{\boldsymbol{x}}+\frac{\tau_{s}^{2}}{(1+\mu)^{2}+\tau_{s}^{2}} \hat{\boldsymbol{y}},
$$

which is dominated by inwards radial drift for smaller particles; $\mathbf{w}_{s} /\left(\eta U_{K}\right) \approx-2 \tau_{s} \hat{\boldsymbol{x}}$ for $\tau_{s} \ll 1, \mu \ll 1$. If the dust is separated from the disc mid-plane, it also drifts towards the mid-plane due to vertical pressure gradients $\partial P / \partial \ln z$, with velocity

$\frac{\mathrm{w}_{s, z}}{\eta U_{K} r} \approx \eta^{-1 / 2} \frac{\tau_{s}}{1+\tau_{s}}$,

which is significantly larger than the radial drift for small particles $\left(\eta^{-1 / 2} \sim r / h \gg 1\right)$ owing to the larger effective vertical gravitational force. Although $\mathbf{w}_{s}$ depends explicitly on the gas pressure gradient (through $\eta$ ), by using $\eta r$ as a length unit and $\Omega$ as a time unit, this dependence drops out, except in the relative size of $\mathrm{w}_{s, z}$ compared to $\mathrm{w}_{s, x}$. Equivalently, the dependence of a linear mode on $\eta$ can be captured by simply rescaling its wavelength by $\eta r$ and its timescales by $\Omega$. We can, thus, ignore the dependence of our results on $\eta$ (equivalently $\partial P / \partial \ln r$ or $h / r$ ) throughout our analysis, so long as we assume that it is relatively small. Note that the local approximation must break down for modes with wavelengths longer than the vertical scale height, $k=|\boldsymbol{k}| \lesssim \eta^{1 / 2}(\eta r)^{-1}$.

\subsection{Equations of motion for the gas and dust}

Although we will not directly solve the fluid equations in this article, it is helpful to present them here in order to highlight the most important terms and their effects. As discussed above, we consider a local patch of disc in a frame moving with the local Keplerian velocity. We also assume the gas to be locally incompressible, which is a good approximation for all modes of interest (see Section 2.2.1 below). The gas equations are then

$\nabla \cdot \boldsymbol{U}=0$

$\frac{\partial}{\partial t} \boldsymbol{U}+\boldsymbol{U} \cdot \nabla \boldsymbol{U}+2 \Omega \hat{z} \times \boldsymbol{U}=-S U_{x} \hat{\boldsymbol{y}}-\frac{\nabla P}{\rho}-\frac{\rho_{d}}{\rho} \frac{\boldsymbol{U}-\boldsymbol{V}}{t_{s}}$,

while the dust satisfies

$\frac{\partial}{\partial t} \rho_{d}+\nabla \cdot\left(\rho_{d} \boldsymbol{V}\right)=0$

$\frac{\partial}{\partial t} \boldsymbol{V}+\boldsymbol{V} \cdot \nabla \boldsymbol{V}+2 \Omega \hat{z} \times \boldsymbol{V}=-S V_{x} \hat{\boldsymbol{y}}-\frac{\boldsymbol{V}-\boldsymbol{U}}{t_{s}}$.

Here, $\boldsymbol{U}$ and $\boldsymbol{V}$ are the local gas and dust velocities, with $S=-(3 / 2) \Omega$, the local Keplerian velocity shear. The velocities are decomposed as $\boldsymbol{U}=S x \hat{\boldsymbol{y}}+\Delta \boldsymbol{U}_{0}+\boldsymbol{u}$ and $\boldsymbol{V}=S x \hat{\boldsymbol{y}}+\Delta \boldsymbol{V}_{0}+\boldsymbol{v}$; i.e. each has a shear contribution $S x \hat{\boldsymbol{y}}$, an equilibrium drift contribution $\Delta \boldsymbol{U}_{0}$ (or $\Delta \boldsymbol{V}_{0}$ ) due to the background pressure gradient (see Section 2.1.2), and a perturbed contribution $\boldsymbol{u}$ (or $\boldsymbol{v}$ ). It is the perturbed velocities $\boldsymbol{u}$ or $v$ that will be considered in our analyses below. The gas density is assumed constant and denoted by $\rho$, while the gas pressure $P$ contains both a perturbed part that is chosen to enforce $\nabla \cdot \boldsymbol{u}=0$, and the equilibrium contribution discussed in Section 2.1.2 (this is balanced by $\Delta \boldsymbol{U}_{0}$ and $\Delta \boldsymbol{V}_{0}$ related terms). The dust density is $\rho_{d}$, while its perturbation is denoted $\delta \rho_{d}=\rho_{d}-\rho_{d 0}$ (where $\rho_{d 0}$ is the mean dust density $\mu=\rho_{d 0} / \rho$ ).

The physical effects that will turn out to be most relevant to the ensuing discussion are as follows:

(i) Dust advection: In the equilibrium frame of the gas (velocity $S x \hat{\boldsymbol{y}}+\Delta \boldsymbol{U}_{0}$ ), the linearized dust continuity equation (6) becomes

$$
\left(\frac{\partial}{\partial t}+\mathbf{w}_{s} \cdot \nabla\right) \frac{\delta \rho_{d}}{\rho_{d 0}}+\nabla \cdot \boldsymbol{v}=0,
$$

showing that the offset in mean velocities between the gas and the dust (equations 2 and 3 ) advects dust-density perturbations with the velocity $\mathbf{w}_{s}$.

(ii) Pressure forces: Any attempt to create motions that compress the gas creates a large pressure force that opposes these motions $(-\nabla P)$. This causes gas motions to be only weakly compressible on the scales of interest, justifying our assumption of incompressibility (see Section 2.2.1). This is not the case for dust motions.

(iii) Coriolis forces: Both the gas and the dust experience Coriolis and velocity shear forces due to the transformation to the rotating, shearing frame. This generates radial from azimuthal velocities, and vice versa, of the form

$$
\begin{aligned}
& \frac{\partial}{\partial t} v_{x}=2 \Omega v_{y}+\ldots, \\
& \frac{\partial}{\partial t} v_{y}=-(2 \Omega+S) v_{x}+\cdots=-\frac{1}{2} \Omega v_{x}+\ldots
\end{aligned}
$$

(likewise for $\boldsymbol{u}$ ). The key effect that enables dust compressions is the generation of radial from azimuthal velocities, which relies only on the Coriolis force. ${ }^{1}$

(iv) Strong drag forces: We focus on the $\tau_{s} \ll 1\left(t_{s} \Omega \ll 1\right)$ regime, where drag forces are strong compared to the $\sim \Omega^{-1}$ time-scales of motions. This means that dust rapidly reaches its 'terminal velocity' (Youdin \& Goodman 2005; Laibe \& Price 2014) where the relative drift velocity of dust compared to gas is determined by the pressure gradient.

(v) Dust-density force (backreaction). The final term in equation (5) is the backreaction of the dust on the gas motions. Because of the mean dust drift $\Delta \boldsymbol{V}_{0}-\Delta \boldsymbol{U}_{0}=\mathbf{w}_{s}$, a local increase or decrease in dust density generates an effective force per unit mass $\boldsymbol{F}$ on the gas (compared to its equilibrium),

$$
\boldsymbol{F}=\left(\rho_{d}-\rho_{d 0}\right) \frac{\mathbf{w}_{s}}{\rho t_{s}}=\mu \frac{\delta \rho_{d}}{\rho_{d 0}} \frac{\mathbf{w}_{s}}{\tau_{s}} \Omega, .
$$

\subsubsection{Justifying the assumption of incompressibility}

A perturbation of wavenumber $k$, with a gas-density variation $\delta \rho / \rho$, will exert a force per unit mass on the surrounding gas of order $\sim$ $k c_{s}^{2}(\delta \rho / \rho)$. Similarly, the force per unit mass exerted due to a drifting

\footnotetext{
${ }^{1}$ In fact, all of the instabilities we consider here continue to operate in a very similar way in the absence of velocity shear, albeit with modified frequencies and growth rates.
} 
dust-density perturbation (the dust-feedback mechanism that enables streaming instabilities; see Section 3.3) is $\sim \mu\left(\mathbf{w}_{s} / \tau_{s}\right)\left(\delta \rho_{d} / \rho_{d 0}\right) \Omega \sim$ $\max (\mu, 1) \eta^{1 / 2} h^{-1} c_{s}^{2}\left(\delta \rho_{d} / \rho_{d 0}\right)$. Here, we have used the scalings from equations (1) and (2), assuming in-plane radial drift $\mathrm{w}_{s, x} /\left(\eta U_{K} r\right)$ $\approx-2 \tau_{s} / \max (\mu, 1)$. Comparison of the two estimates shows that a gas-density perturbation $\delta \rho / \rho \sim \max (\mu, 1) \eta^{1 / 2}(k h)^{-1}\left(\delta \rho_{d} / \rho_{d 0}\right)$ will affect the gas with similar force to the dust perturbation $\delta \rho_{d} / \rho_{d 0}$. Since $\max (\mu, 1) \eta^{1 / 2}(k h)^{-1} \ll 1$, we see that only very small gasdensity perturbations are needed to create pressure forces similar to the dust backreaction force that drives the instability, showing that the gas can be very well approximated as incompressible. ${ }^{2}$

More explicitly, both Paper I and Youdin \& Johansen (2007) showed through numerical and analytic calculations of the dispersion relation that allowing or removing compressibility has a negligible effect on the behaviour of the streaming-instability. Note that the toy secular instability of Goodman \& Pindor (2000) requires compressibility of the gas surface density, so is not described by our models.

\section{RESONANT FORCING OF LINEAR SYSTEMS}

In this section, we discuss heuristically how instabilities of the coupled dust-gas system arise from the resonant driving of the dustdensity perturbations by the gas, coupled with resonant driving of gas modes by the dust. The discussion tells us that in order to understand the mechanisms for the streaming and settling instabilities, we should consider how these resonant driving mechanisms feed back on each other. If the dust perturbation generated by a gas mode has the effect of resonantly forcing this original mode, the coupled system will be linearly unstable.

\subsection{Secular growth at resonance}

It is well known that applying a time-varying force to an oscillator system leads to a particularly strong response when the frequency of the forcing matches that of a natural mode of the system. As a particularly simple example, the system

$\partial_{t} f(t)+i \omega f(t)=F \mathrm{e}^{-\mathrm{i} \omega_{F} \mathrm{t}}, \quad f(0)=0$,

has the (bounded) solution $f(t)=i F\left(\omega-\omega_{F}\right)^{-1}\left(\mathrm{e}^{-\mathrm{i} \omega \mathrm{t}}-\mathrm{e}^{-\mathrm{i} \omega_{F} \mathrm{t}}\right)$ for off-resonant $\left(\omega \neq \omega_{F}\right)$ forcing, while resonant forcing $\left(\omega=\omega_{F}\right)$ leads to (unbounded) continuous secular growth, $f(t)=F t \mathrm{e}^{-\mathrm{i} \omega \mathrm{t}}$. In the more complex case where $f(t) \rightarrow \boldsymbol{f}(t)$ is a system of variables, $i \omega \rightarrow i \boldsymbol{\Omega}$ represents a general linear operator and $F \rightarrow \boldsymbol{F}$ is a vector of forcing amplitudes, the strength of the effect of forcing at resonance is governed by $\boldsymbol{\xi}^{L} \cdot \boldsymbol{F}$, where $\boldsymbol{\xi}_{L}$ is the left eigenvector of $i \boldsymbol{\Omega}$ that is associated with the frequency of the forcing $\left(\omega_{F}\right)$. In other words, the system will execute unbounded secular growth $f(t) \propto t \mathrm{e}^{-\mathrm{i} \omega t}$ so long as two conditions are met: (i) The forcing frequency $\omega_{F}$ matches an eigenfrequency $\omega$ of $i \boldsymbol{\Omega}$; and (ii) The left eigenmode (termed $\xi^{L}$ ) of $i \boldsymbol{\Omega}$ associated with $\omega=\omega_{F}$ is not orthogonal to the forcing direction, $\boldsymbol{\xi}^{L} \cdot \boldsymbol{F} \neq 0$. Physically, the idea is that in order to resonantly drive a given eigenmode of the system, the applied forcing must both match its frequency and lie along a direction that is able to force the motions involved in the mode. ${ }^{3}$

\footnotetext{
${ }^{2}$ In the settling instability, where $\mathrm{w}_{s, z} \sim \eta^{-1 / 2} \mathrm{w}_{s, x}$, the factor becomes $\sim \max (\mu, 1)(k h)^{-1}$, but this is still much smaller than unity for all modes considered.

${ }^{3}$ Note that in the $1 \times 1$ system of equation (11), $\xi^{L}=1$, so condition (ii) is always met if $F \neq 0$.
}

The RDI theory of Squire \& Hopkins (2018a) quantifies how such resonant interactions lead to linear instabilities of coupled linear systems. The relation of their result to the above arguments can be understood by considering a system such as

$$
\begin{gathered}
\partial_{t} \boldsymbol{f}_{1}+i \boldsymbol{\Omega}_{1} \cdot \boldsymbol{f}_{1}=\boldsymbol{F}_{2 \rightarrow 1}, \\
\partial_{t} \boldsymbol{f}_{2}+i \boldsymbol{\Omega}_{2} \cdot \boldsymbol{f}_{2}=\boldsymbol{F}_{1 \rightarrow 2},
\end{gathered}
$$

where the homogenous part of system $1\left(\partial_{t} \boldsymbol{f}_{1}+i \boldsymbol{\Omega}_{1} \cdot \boldsymbol{f}_{1}=0\right)$ represents the linearized equations of motion of gas in the absence of dust, while the homogenous part of system $2\left(\partial_{t} \boldsymbol{f}_{2}+i \boldsymbol{\Omega}_{2} \cdot \boldsymbol{f}_{2}=0\right)$ represents the motion of dust in the absence of gas. The forcing terms, $\boldsymbol{F}_{2 \rightarrow 1}$ and $\boldsymbol{F}_{1 \rightarrow 2}$ represent the forcing of gas motions by dust motions and the forcing of dust motions by gas motions, respectively. RDI theory states that resonant instabilities arise from the effect of system one forcing system two $\left(\boldsymbol{\xi}_{2}^{L} \cdot \boldsymbol{F}_{1 \rightarrow 2}\right)$ multiplied by the effect of system two forcing system one $\left(\boldsymbol{\xi}_{1}^{L} \cdot \boldsymbol{F}_{2 \rightarrow 1}\right.$; see equation 4 of Squire \& Hopkins 2018a). ${ }^{4}$ If both of these effects are strong $\left(\boldsymbol{\xi}_{2}^{L} \cdot \boldsymbol{F}_{1 \rightarrow 2} \neq 0\right.$ and $\left.\xi_{1}^{L} \cdot \boldsymbol{F}_{2 \rightarrow 1} \neq 0\right)$, the coupled system will exhibit exponential growth, so long as the two are not perfectly out of phase. This makes intuitive sense: A small excitation of the first eigenmode will cause a particularly strong response in the second, which in turn causes a strong response in the first, thus leading to runaway growth of the coupled modes.

In the next two subsections and Figs 2 and 3, we apply this idea to the coupled dust-gas system in a disc. These ideas are then used in Sections 4-6 to explain the operation of the streaming and settling instabilities.

\subsection{The influence of gas motions on the dust}

Based on the ideas laid out in the previous paragraphs, let us consider the effect of a gas epicycle on the dust. This section is intended to be read in conjunction with Fig. 2, which sketches the relevant ideas.

We are interested in gas epicycles, which arise due to the Coriolis force and local shear (see equation 9), and have frequency $\omega= \pm \hat{k}_{z} \Omega= \pm\left(k_{z} / k\right) \Omega$ for axisymmetric motions. The $k_{z} \neq 0$ requirement for oscillations occurs because of the incompressibility condition, which implies that the radial motions generated by azimuthal motions must also be accompanied by vertical motions. The structure of the mode, shown in Fig. 2, involves in-phase radial and vertical motions with azimuthal velocity perturbations $\pi / 2$ out of phase (either leading or trailing, depending on the sign of $\omega$ ).

To lowest order in $\tau_{s}$, the dust simply follows the gas motions. However, in order to cause dust-density perturbations, deviations of the dust bulk velocity from that of gas are necessary because the gas is incompressible. The strongest of these deviations is caused by the Coriolis force on the dust azimuthal velocity, which generates a radial dust velocity $\partial_{t} v_{x}=2 \Omega v_{y}$. In an epicycle, the $u_{y}$ perturbation generates an azimuthal dust velocity perturbation $\left(v_{y}\right)$ through drag, which in turn creates a radial dust-velocity perturbation $v_{x}$. Because this $v_{x}$ generated by the Coriolis force is not accompanied by vertical dust motions (as is the case for the $v_{x}$ caused directly by the epicycle), it is compressive, $\nabla \cdot \boldsymbol{v} \neq 0$ and generates a dust-density perturbation $\delta \rho_{d}$. In the absence of dust backreaction, this forcing of the dustdensity perturbation will cause it to grow secularly in time if the dust's natural frequency matches that of the epicycle. This frequency is determined by the advection of the dust at velocity $\mathbf{w}_{s}$ and the lack of a dust pressure response, which implies that the dust mode's natural

\footnotetext{
${ }^{4}$ In the notation of Squire \& Hopkins (2018a), system 2 is $A$, system 1 is $F$,
} $F_{2 \rightarrow 1}=T_{\mathrm{FA}}^{(1)} \cdot \xi_{A}^{R}$ and $F_{1 \rightarrow 2}=C \cdot \xi_{F}^{R}$. 


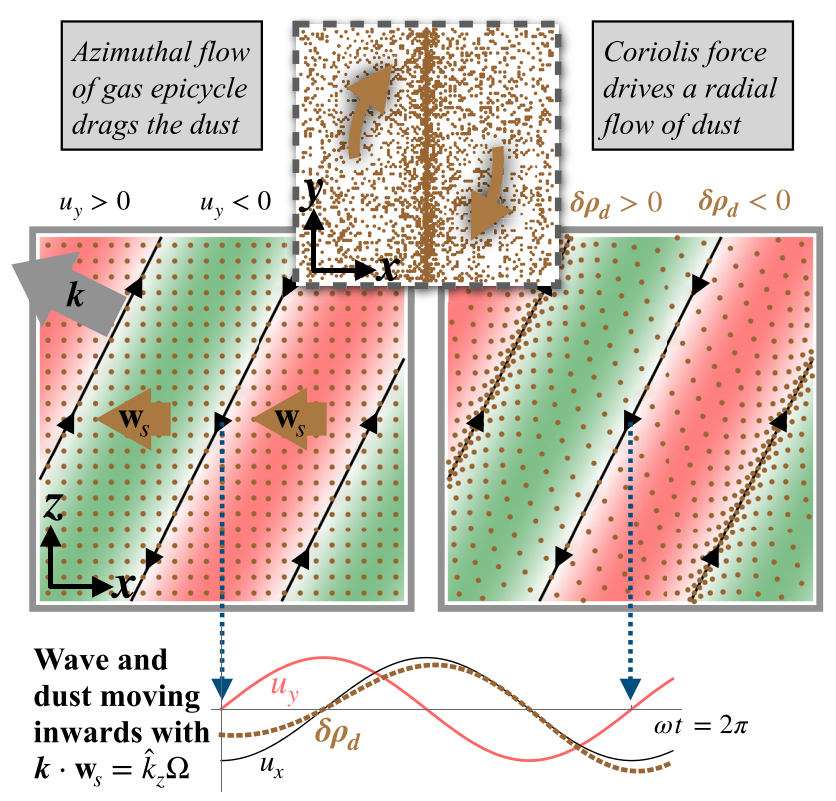

Figure 2. Response of the dust to a gas epicyclic perturbation. The structure of a positive-frequency gas epicycle, which propagates in the $\hat{\boldsymbol{k}}$ direction, is shown in the left-hand panel. Lines show the $u_{x}$ and $u_{z}$ flow lines (crests), colours show $u_{y}$ perturbations (red $u_{y}>0$, green $u_{y}<0$ ). We see that $u_{x}$ lags $u_{y}$ by $\pi / 2$ as the wave moves inwards (leftwards). The $u_{y}$ perturbations cause in-phase $v_{y}$ perturbations due to the drag force, which then generate $v_{x}$ perturbations due to the Coriolis force (top sub panel, which is centred on a maximum of $u_{x}$ and $u_{z}$ ). This compressive $v_{x}$ perturbation generates a dust-density perturbation between the regions of large $v_{y}$. If the natural dust frequency $\omega=\boldsymbol{k} \cdot \mathbf{w}_{s}$ matches that of the epicycle, dust-density perturbations grow secularly in time, with density maxima in phase with $u_{x}$ maxima. The bottom panel illustrates qualitatively the time evolution of a point in the middle of the domain (see blue-dashed lines) over one wave period, showing the secular growth of dust-density perturbations $\delta \rho_{d} \propto t \cos (\omega t)$.

frequency is simply that of bulk motion of a static perturbation, $\omega=$ $\boldsymbol{k} \cdot \mathbf{w}_{s}$ (in the gas frame). Thus, an epicycle for which $\boldsymbol{k} \cdot \mathbf{w}_{s}= \pm \hat{k}_{z} \Omega$ will excite strong dust-density perturbations, which grow secularly in time, with the density maxima in phase with the gas $u_{x}>0$ perturbations.

For a more rigorous description of the above, it is helpful to write down the velocity components of the left eigenmode $\xi_{D}^{L}$ associated with a dust-density perturbation, which is straightforwardly derived from equations (6) and (7) (see Paper I) as ${ }^{5}$

$$
\begin{aligned}
\xi_{D, v}^{L} & =\left(v_{x}, v_{y}, v_{z}\right) \\
& =t_{s}\left(-i k_{x}, 0,-i k_{z}\right)+t_{s}\left(i \frac{k_{x} \tau_{s}^{2}}{1+\tau_{s}^{2}},-i \frac{2 k_{x} \tau_{s}}{1_{+} \tau_{s}^{2}}, 0\right) .
\end{aligned}
$$

Forcing the dust in a direction parallel to $\xi_{D, v}^{L}$ generates a strong dust-density perturbation, while forcing perpendicular to $\xi_{D, v}^{L}$ does not cause a dust-density perturbation (see Section 3.1). The first term of equation (13), which is the lowest order response in $\tau_{s}$, is simply a compressive motion, stating (as expected) that driving a direct compression results in a strong dust-density perturbation. This term is perpendicular to the incompressible velocity field of the gas epicycle, and thus does not contribute to the dust-density response. The $y$ component of the second term relates to the discussion above; a dust-

${ }^{5}$ Note that of $\xi_{D, v}^{L}$ has units of inverse velocity to give a dimensionless quantity when dotted with the right eigenmode, viz., it is a covector.

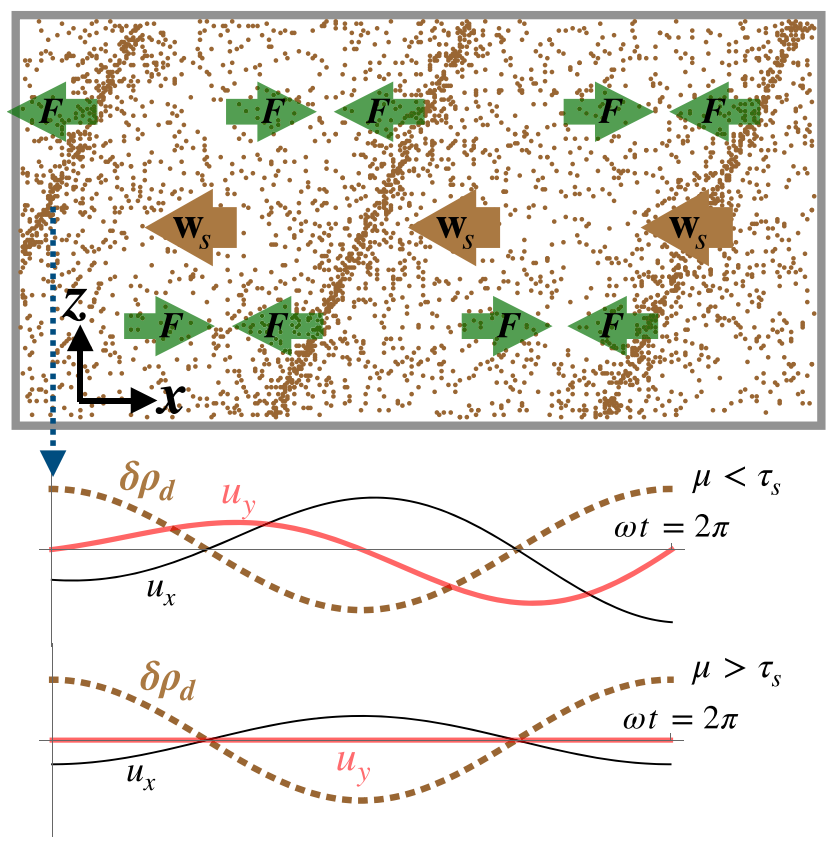

Figure 3. Response of (incompressible) gas to a dust-density perturbation. Local dust-density perturbations produce a variable force $\boldsymbol{F}$ on the gas in the direction $\delta \rho_{d} \mathbf{w}_{s}$, because the mean dust drift $\mathbf{w}_{s}$ leads to the dust-densitydependent back-reaction force $\boldsymbol{F}=\delta \rho_{d} \mathbf{w}_{s} / \tau_{s}$. This is shown with green arrows in the top panel. This periodic forcing with frequency $\boldsymbol{k} \cdot \mathbf{w}_{s}$ excites gas epicycles if $\mu \ll \tau_{s}$, which will grow secularly if their frequency matches that of the forcing (top time-trace panel). If $\mu \gg \tau_{s}$, the forcing balances the drag force, directly driving a velocity in the direction $\boldsymbol{F} \propto \delta \rho_{d} \mathbf{w}_{s}$ (bottom time-trace panel). When $\mathbf{w}_{s}$ is parallel to $\boldsymbol{k}$, no gas motions can be excited because the gas pressure force (incompressibility) directly opposes the dust forcing.

density perturbation can be caused by forcing a radially dependent azimuthal velocity. It is straightforward to verify that this term relies on the Coriolis force on the dust to be non-zero. The $x$-component of the second term is yet higher order in $\tau_{s}$ and will be discussed in Section 4.

\subsection{The influence of dust perturbations on the gas}

The excitation of gas motions due to dust-density perturbations is a simpler process. As discussed in point (v) of Section 2.2 (equation 10), because of the mean dust-gas drift $\mathbf{w}_{s}$, a dust perturbation causes an effective force per unit mass $\boldsymbol{F}=\mu \delta \rho_{d} \mathbf{w}_{s} / t_{s}$ on the gas (compared to the equilibrium, in which the dust force is balanced by a slight change in gas velocity; Nakagawa et al. 1986). This force is in the $\mathbf{w}_{s}$ direction for an overdensity, or in the $-\mathbf{w}_{s}$ direction for an underdensity. Because the dust is advected with velocity $\mathbf{w}_{s}$, this amounts to a periodic forcing of the gas velocity in the direction $\pm \mathbf{w}_{s}$ with frequency $\omega=\boldsymbol{k} \cdot \mathbf{w}_{s}$. This is sketched in Fig. 3 .

The details of the gas response depend on the dust-to-gas ratio $\mu$. If $\mu \ll \hat{k}_{z} \Omega t_{s}=\hat{k}_{z} \tau_{s}$, gas epicycles are excited, but not modified appreciably by the dust, because the drag force is too small. In contrast, if $\mu \gg \hat{k}_{z} \tau_{s}$, the Coriolis forces become subdominant and relatively unimportant for the gas motion, because the drag dominates. In the former case, the system is nearly identical to the forced oscillator of equation (11); the dust drives epicycles, which grow secularly in time if their frequency matches that of the dust driving. Just as in equation (11), the phase of forcing $\left(\boldsymbol{F} \propto \delta \rho_{d} \mathbf{w}_{s}\right)$ 


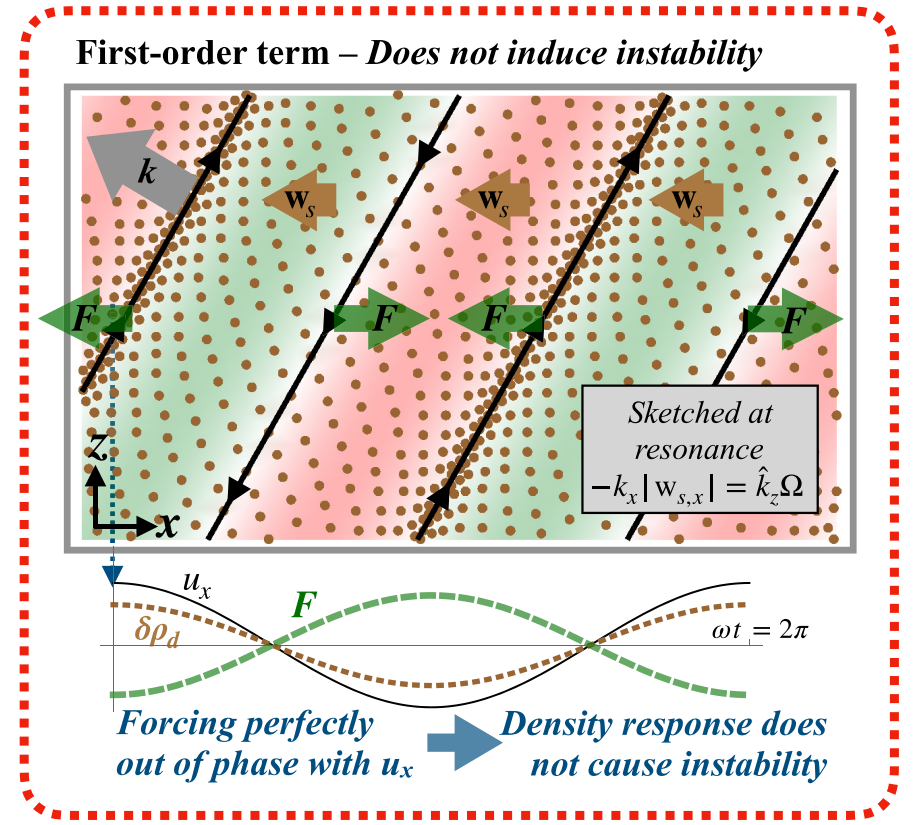

\section{Second-order term - Drives instability \\ Relies on reduction of radial dust flow by Coriolis/shear}

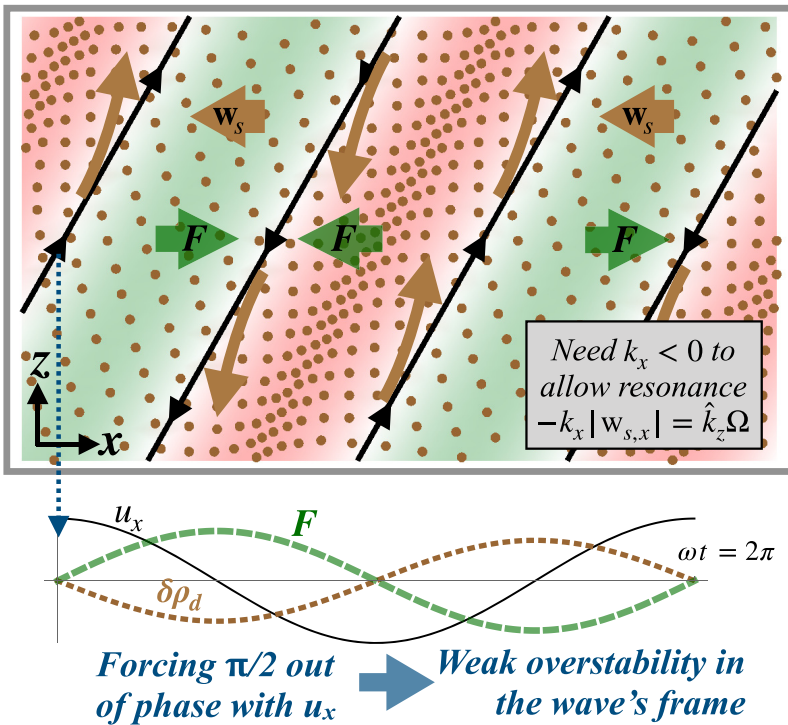

Figure 4. The low-dust-to-gas-ratio $(\mu<1)$ streaming instability. The left-hand panel illustrates how the dust feedback sketched in Figs 2 and 3 fails to cause an instability: The force $(\boldsymbol{F})$ from the dust-density perturbation $\left(\delta \rho_{d}\right)$ generated by a gas epicycle is exactly out of phase with the force that most strongly amplifies the epicycle. The right-hand panel shows the weaker interaction that does lead to instability: Deflection by the Coriliois force manifests itself as a slight decrease in the magnitude of $v_{x}$, which causes a dust compression between maxima of $\left|u_{x}\right|$. The velocity deflection of the dust that leads to this compression (see equation 13) is illustrated with the brown arrows. The $\pi / 2$ offset between $u_{x}$ and $\boldsymbol{F}$ generates an overstability in the wave frame. As in Fig. 2, the bottom panels illustrate the time evolution of gas velocity $\left(u_{x}\right)$, dust density $\left(\delta \rho_{d}\right)$, and dust-density-induced force $(\boldsymbol{F})$ perturbations as the wave propagates in the $\boldsymbol{k}$ direction, at the point in each domain shown by a blue arrow.

matches that of the forced velocity component $\left(\boldsymbol{u} \cdot \mathbf{w}_{s}\right) /\left|\mathbf{w}_{s}\right|$. In the latter limit, where drag forces dominate, the gas velocity rapidly (faster than $\sim\left(\hat{k}_{z} \Omega\right)^{-1}$ ) reaches balance between the forcing and the drag. This again leads to a gas response that is in phase with the forcing from the density, although there is now no secular response at resonance and the gas responds to all driving frequencies in a similar way. In practice, the most significant difference between the gas responses in the two regimes is whether or not an appreciable azimuthal gas velocity is excited by radially or vertically streaming dust: If $\mu \ll \tau_{s}$, an azimuthal velocity $u_{y}$ is excited because gas motions are essentially epicycles; if $\mu \gg \tau_{s}$, the $u_{y}$ response is weak because the Coriolis forces are weaker than the dust-driving forces. Nonetheless, because the phase of the $u_{x}$ and $u_{z}$ responses remains the same in the $\mu \ll \tau_{s}$ and $\mu \gg \tau_{s}$ regimes, the character of the streaming and settling instabilities remains nearly independent of $\mu / \tau_{s}$.

\subsubsection{The necessity of two-dimensional motions}

A dust-density perturbation for which $\boldsymbol{k}$ and $\mathbf{w}_{s}$ are exactly parallel $\left(\boldsymbol{k} \times \mathbf{w}_{s}=0\right)$ will not excite any gas motions if the gas is truly incompressible. This is because $\boldsymbol{F}$ (the dust backreaction force on the gas) is parallel to $\boldsymbol{k}$ so is purely compressive, and is therefore entirely resisted by the assumed infinitely strong pressure forces (see Section 2.2.1). As a consequence, for radial streaming, a purely radial dust-density perturbation cannot excite gas motions. In the opposite case of $\boldsymbol{k} \cdot \mathbf{w}_{s}=0$ ( $k_{x}=0$ with radial streaming), the forcing is perpendicular to the motions required to drive the dust-density perturbation, so cannot excite an instability. This explains why the traditional mid-plane streaming instability (with $\mathbf{w}_{s} \propto \hat{x}$ ) cannot operate with a purely one-dimensional perturbation; it requires $k_{x}$ $\neq 0$ and $k_{z} \neq 0$.

With compressive gas perturbations, the above argument does not hold and fast-growing one-dimensional instabilities can indeed occur (Hopkins \& Squire 2018). However, in order to drive an instability, a gas compression created by a dust perturbation must not 'outrun' the dust perturbation, since this would halt the feedback loop necessary for instability. Because gas compressions propagate at the sound speed, such instabilities require dust drift velocities that approach the sound speed, which can never occur in dense regions of protoplanetary discs.

\section{THE LOW- $\mu$ STREAMING INSTABILITY}

Through Section 3, we have seen that our understanding of instabilities should be guided by how the dust-density perturbations generated by a gas epicycle feed back on this epicycle. In this section, we apply these ideas to low-dust-to-gas-ratio $(\mu<1)$ dust moving primarily in the negative radial direction $\left(\mathbf{w}_{s} \propto-\hat{\boldsymbol{x}}\right)$, as appropriate to the disc mid-plane. This is the 'low- $\mu$ streaming instability.' Unfortunately, it transpires that its mechanism is more complex than that of the settling instability (which involves vertically streaming dust, $\mathrm{w}_{s, z}$ $\neq 0$ ), because the dust and gas responses sketched in Figs 2 and 3 happen to be exactly out of phase. We choose to present this case first because it has been much better studied in previous literature, but suggest that it may be helpful to a reader to consider Section 5 concurrently. Helpful discussion can also be found in Zhuravlev (2019; specifically in their Section 4).

The physics of the streaming instability is sketched in Fig. 4. Note that we are implicitly assuming that the illustrated wave satisfies the resonance condition $\boldsymbol{k} \cdot \mathbf{w}_{s}=\hat{k}_{z} \Omega$, so that the resonant interactions 
sketched in Figs 2 and 3 are applicable. The most important feature, shown in the left-hand panel of Fig. 4, is that the resonant excitation of dust-density perturbations by the epicycle's azimuthal velocity (Fig. 2) has the wrong phase to cause an instability (the red border is used to emphasize this point). More specifically, the interaction produces dust maxima in phase with $u_{x}$ maxima, as shown in Fig. 2, while the force from a dust maximum (minimum) is in the $-\hat{\boldsymbol{x}}$ $(+\hat{\boldsymbol{x}})$ direction (see equation 10). As shown in Fig. 3, this force is exactly opposite to that which most strongly forces the gas, and thus does not cause an instability (in fact, any other phase would cause instability). In RDI theory, this phase anti-alignment manifests itself mathematically as a purely real perturbation to the frequency of the epicycle at the lowest order in $\tau_{s}$ (see equation 34 of Paper I).

The right-hand panel sketches the weaker dust feedback that does lead to instability. To the lowest order, the dust follows the incompressible streamlines of the gas in the $x-z$ plane; however, due to the Coriolis forces, a small part of the dust's radial velocity is deflected into the azimuthal direction. This deflection decreases the magnitude of $v_{x}$, making the flow of dust slightly compressive, and thus concentrating dust between regions of negative and positive $u_{x}$ (for a $k_{x}>0$ wave resonating with a $\omega<0$ epicycle, the phase of $\delta \rho_{d}$ is reversed). The forcing of the gas induced by this dustdensity perturbation is now $\pi / 2$ out of phase with the optimal driving illustrated in Fig. 3, which is sufficient to render the epicycle unstable, causing an overstability in the wave's frame because of the $\pi / 2$ phase shift. Mathematically, this interaction is described by the $x$ component of the of the second term in equation (13) and is one order higher in $\tau_{s}$ than the compression caused by the azimuthal velocity. Because this term is one order higher in $\tau_{s}$, it is outside the "terminal velocity approximation' for dust (see Zhuravlev 2019 and point iv of Section 2). At the same order, there is also a contribution from the azimuthal relative dust drift $\mathrm{w}_{s, y}$, which can modify the growth rate somewhat (Paper I).

\subsection{Model summary}

Our model qualitatively explains some key features of the streaming instability, when $\mu<1$, as follows:

(i) Its fastest growing modes are those that satisfy the epicycle resonant condition $\boldsymbol{k} \cdot \mathbf{w}_{s}=\hat{k}_{z} \Omega$, where the effect of gas on dust, and vice versa, is strong. This idea is explored quantitatively in Paper I.

(ii) The growth rate of the low- $\mu$ streaming instability decreases with $\tau_{s}$. This is not simply because small dust grains are "better coupled' to the gas, as often stated - indeed better-coupled dust interacts with higher frequency motions (which might naively be associated with higher growth rates). Rather, it is because the geometry of the system with purely radial streaming causes the normal leading-order dust backreaction force on the gas (equation 10) to be out of phase with the gas motions of an epicycle. The feedback term that causes the instability is therefore the next-order term in $\tau_{s}$, thus causing the growth rate to decrease with grain size. Equivalently, the feedback that drives the instability is not contained within the 'terminal velocity approximation' (see Zhuravlev 2019).

(iii) The streaming instability can operate only if two-dimensional motions are allowed: If $k_{z}=0$, not only does the gas epicycle have zero frequency (and thus cannot resonate with the streaming dust), but dust perturbations cannot feed back to excite incompressible gas motions if $\mathbf{w}_{s}$ and $\hat{\boldsymbol{k}}$ are parallel. The general conclusion is unchanged if one allows for finite gas compressibility (see Section 3.3.1). (iv) Dust perturbations are approximately $\pi / 2$ out of phase with those of $u_{x}$ and $u_{z}$, and thus in phase with $\pm u_{y}$ (with the sign depending on the sign of $k_{x}$ ).

\section{THE SETTLING INSTABILITY}

We now apply the same analysis as above to the case where dust is settling towards the mid-plane, with a drift velocity $\left|\mathrm{w}_{s, z}\right| \gg\left|\mathrm{w}_{s, x}\right| \gg$ $\left|\mathrm{w}_{s, y}\right|$. This leads to the 'settling instability' of Paper I. As mentioned above, the situation is simpler in this case than for the streaming instability because there is no longer a phase anticorrelation between the dust feedback and the epicycle. Properties of the settling instability are explored extensively in Zhuravlev (2019).

As in Section 4, our first assumption is that the resonance condition is satisfied

$k_{x} \mathrm{~W}_{s, x}+k_{z} \mathrm{~W}_{s, z}= \pm \hat{k}_{z} \Omega$,

such that there are strong interactions between the gas and dust modes. In order to use the same $\omega>0, k_{x}<0, k_{z}>0$ convention as in Figs 2-4 for illustration, let us take $\mathrm{w}_{s, z}>0$, as applicable below the disc mid-plane. We sketch this case in the left-hand panel of Fig. 5. The dust-density perturbation generated by the epicycle is again in phase with $u_{x}$ as shown in Fig. 2; however, the force on the gas, which is proportional to $\delta \rho_{d} \hat{\mathbf{w}}_{s}$, is now primarily in the $+\hat{z} \delta \rho_{d}$ direction. Although the $x$-directed force is again out of phase with that needed to drive the epicycle, the stronger $z$-directed force is in phase with $u_{z}$, and thus will strongly drive the epicycle (see Fig. 3).

Modes with the opposite $k_{x}$ (for the same sign of $\mathrm{w}_{s, z}$ ) feel an even stronger feedback from the dust, because the dust forcing on the epicycle can be in phase with both $u_{x}$ and $u_{z}$ (see lower right-hand panel of Fig. 5). Such modes have somewhat smaller wavelengths because the $k_{x} \mathrm{w}_{s, x}$ and $k_{z} \mathrm{~W}_{s, z}$ have opposite signs, thus increasing the wavenumber $k(k=|k|)$ required to satisfy equation (14). A particularly extreme version - in which $\mathbf{w}_{s}$ is nearly aligned with the wavefronts - is sketched in the right-hand panel of Fig. 5. This was termed the 'double-resonant mode' in Paper I. We see that as the epicycle propagates (to the right and up now, because $k_{x}>0$ ), the force from the dust perturbation that it generates is very close to the direction of the incompressible gas velocity, causing a particularly strong response and a fast growing mode. In fact, as shown in Paper I, the growth rate of this mode approaches infinity as $k \rightarrow \infty$ for any $\tau_{s}$ and any $\mu\left(\omega \propto k^{1 / 3} \mu^{1 / 3} \tau_{s}^{1 / 3}\right)$, surpassing $\Omega$ for sufficiently small wavelengths (note that $\hat{\boldsymbol{k}} \cdot \mathbf{w}_{s} \rightarrow 0$ for this mode, so the resonance condition 14 implies that the mode must be at high $k$ ). As it moves to shorter wavelengths, the wave loses its epicyclic character, although the basic phase structure remains similar, with $\delta \rho_{d}$ being primarily driven by azimuthal velocities and the Coriolis force.

\subsection{Model summary}

Our model qualitatively explains some of the key features of the settling instability as follows:

(i) Unlike the streaming instability, its maximum growth rate does not depend on $\tau_{s}$ and is just as large for arbitrarily small grains (although the characteristic resonant wavelength decreases with smaller $\tau_{s}$ ). The reason is that the dust feedback, which forces the fluid in the direction $\delta \rho_{d} \hat{\mathbf{w}}_{s}$, is partially in phase with the velocity of the epicycle. This implies that the leading-order dust backreaction force, which enables the instability, is independent of $\tau_{s}$, causing a $\tau_{s}$-independent growth rate. 


\section{Approaching the double-resonant angle}
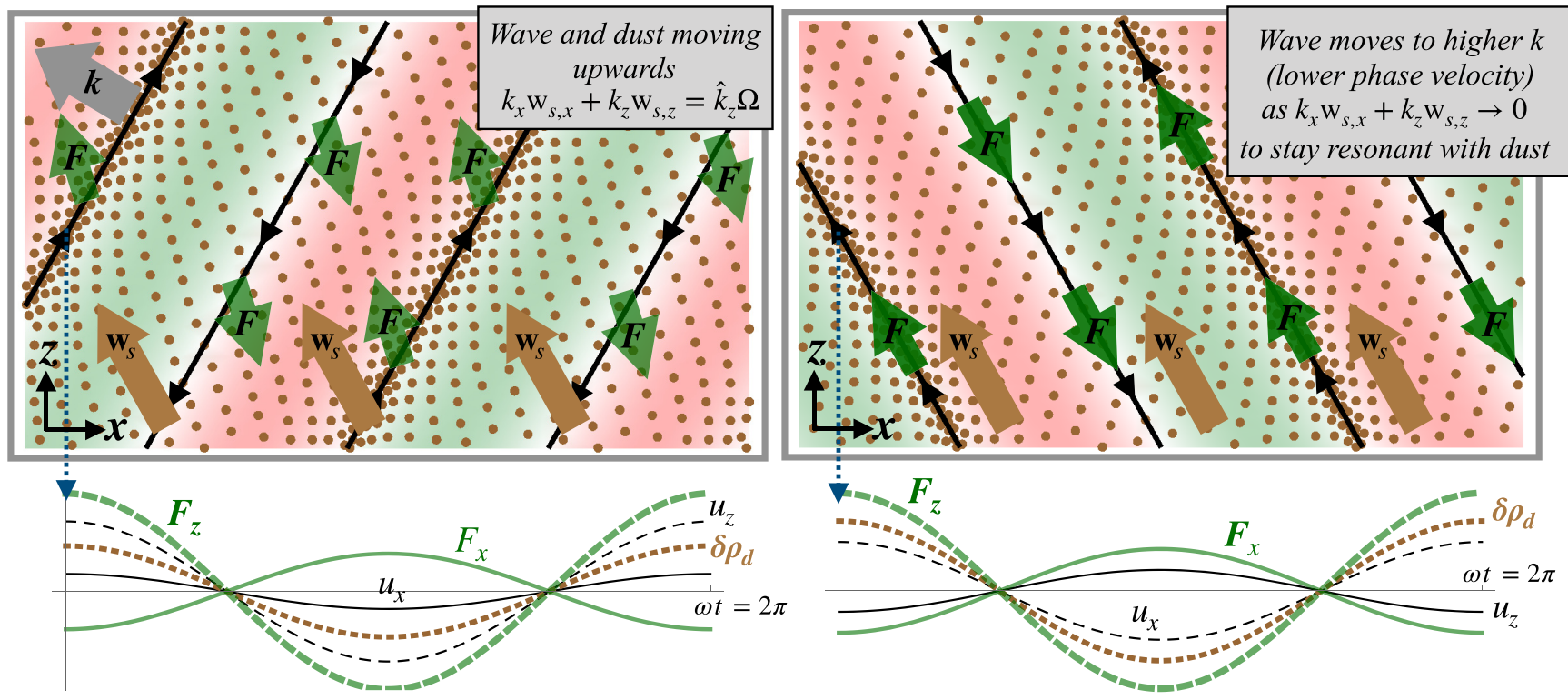

Figure 5. The settling instability. The left-hand panel illustrates the fundamental difference in dust-gas interaction that arises when the dust also streams vertically: Even though the radial velocity is forced out of phase by the dust backreaction $\boldsymbol{F}$ (as for the streaming instability; Fig. 4), the forcing of the vertical gas velocity by the dust is now in phase. The resulting instability is much stronger than the streaming instability when $\tau_{s}<1$, because it relies on the stronger azimuthal-velocity-induced dust feedback (Fig. 2). The right-hand panel illustrates why the 'double-resonant' mode of Paper I is particularly fast growing: The forcing of the epicycle by the gas is aligned and in phase with both $u_{x}$ and $u_{z}$, generating a particularly strong gas response that leads to growth rates larger than $\Omega$ at sufficiently short wavelengths (Paper I). The lower panels illustrate time traces of gas velocity $\left(u_{x}, u_{z}\right)$, dust density $\left(\delta \rho_{d}\right)$, and dust backreaction force $\left(F_{x}, F_{z}\right)$ perturbations as the wave propagates in the $\boldsymbol{k}$ direction, at the point in the domain shown with the blue-dotted arrow (the dust is drifting nearly vertically with $\left.\boldsymbol{k} \cdot \mathbf{w}_{s}=\hat{k}_{z} \Omega\right)$

(ii) For the same $\tau_{s}$, the settling instability operates at larger wavelengths than the streaming instability, because the vertical settling drift is larger than the radial drift in a thin disc (Paper I).

(iii) Modes for which the sign of $k_{x} \mathrm{w}_{s, x}$ is opposite to that of $k_{z} \mathrm{~W}_{s, z}$ grow faster than those with the opposite polarity, because the dust forcing is in phase with both $u_{x}$ and $u_{z}$ perturbations. (These modes have smaller wavelengths, however, compared to resonant modes for which $k_{x}$ and $k_{z}$ have the same sign.)

(iv) The 'double-resonant mode,' which has very large $(>\Omega)$ growth rates at small scales, occurs because the forcing of the mode by $\delta \rho_{d}$ perturbations (in the direction $\boldsymbol{F} \propto \delta \rho_{d} \mathbf{w}_{s}$ ) aligns with the incompressible flow velocity, thus driving the gas motions particularly efficiently.

(v) The settling instability is strongly suppressed (similar to the streaming instability) if $\boldsymbol{k}$ and $\mathbf{w}_{s}$ are parallel or, more generally, if $k_{z} / k_{x} \leq \mathrm{w}_{s, z} / \mathrm{w}_{s, x}$. In this case, the forcing is perpendicular to that required for incompressible motions (or out of phase), so only the weaker higher-order dust feedback of the streaming instability is effective at amplifying the epicycle (see Fig. 4, right-hand panel).

\section{THE HIGH- $\mu$ STREAMING INSTABILITY}

A key feature of the streaming instability - noted in Youdin \& Goodman (2005) and Youdin \& Johansen (2007) and studied in more detail in Appendix A of Paper I - is a sudden change in its growth rate and other properties as the dust-to-gas ratio $\mu$ increases beyond unity. The transition, which is illustrated in Fig. 6 and occurs for any $\tau_{s} \lesssim 1$, is likely a key feature of the streaming instability's nonlinear development, since simulations seem to show that the presence of high- $\mu$ regions in the mid-plane is a necessary requirement for the formation of gravitationally bound planetesimals. We discuss this 'high- $\mu$ streaming instability' in this section, illustrating how it operates, why it grows so much more rapidly than the $\mu<1$ streaming instability, and why it appears only for $\mu \geq 1$. As a reminder, our discussion here will not be mathematically rigorous, rather focusing on providing intuitive insight. We will, however, occasionally use expressions from Appendix A of Paper I for clarity. Similarly, Fig. 6 illustrates streaming instability growth rates calculated from a full (numerical) solution to the dispersion relation.

Note that, unlike the streaming instability, there is not a sudden change in the properties of the settling instability as $\mu$ surpasses unity, although its growth rate does keep increasing with $\mu$ with fast-growing modes that resemble the double-resonant mode. For this reason, and because it seems less astrophysically relevant (dust is unlikely to reach such high densities outside of the mid-plane in discs) we do not discuss the settling instability at $\mu>1$.

\subsection{Out-of-phase forcing of the epicycle}

In Section 4 and Fig. 4, we saw that if the dust is streaming radially, the dust-density perturbation induced by an epicycle is almost perfectly out of phase with the perturbation needed to create a strong response from the gas. Only a much weaker (higher order in $\tau_{s}$ ) dust response feeds back to the gas and causes instability, explaining the decrease in streaming instability growth rate with $\tau_{s}$. Here, we discuss how the lowest order feedback does, nonetheless, have important effects: It is responsible for the streaming instability's character change at $\mu=1$. 


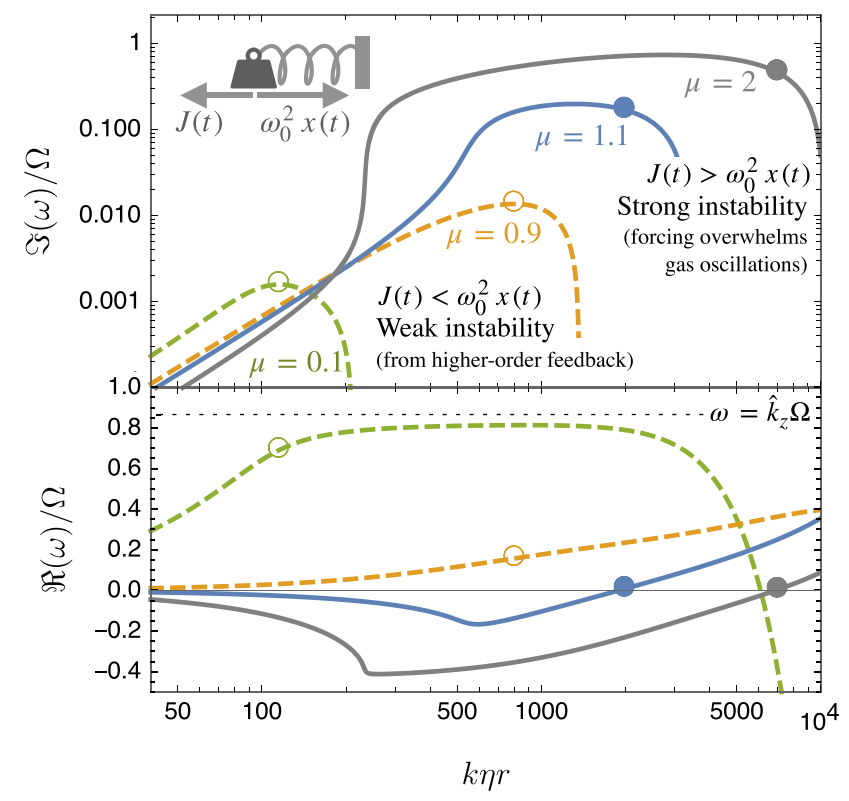

Figure 6. Illustration of the sudden change to the streaming instability at $\mu$ $=1$. We show the growth rate (upper panel) and oscillation frequency (lower panel) of modes as a function of wavelength, for $\tau_{s}=0.01$ and $\mu=0.1$, $0.9,1.1$, and 2. Each curve is calculated from the full dispersion relation as a function of $k \eta r$ (see Paper I), with a mode angle of $30^{\circ}\left(k_{z} / k_{x}=\sqrt{3}\right.$; other mode angles have similar behaviour). At $\mu<1$, shown with dashed curves, the resonant (fastest-growing) wavelength of the streaming instability is effectively an epicycle, modified by the force $\boldsymbol{F}$ on the gas from the dust. Its real oscillation frequency decreases steadily from its unperturbed value $\left(\omega=\left(k_{z} / k\right) \Omega\right)$ as $\mu$ increases (unfilled circles label the resonant $k$ and $\omega$ in both panels). At $\mu>1$, shown with solid curves, the dust forcing overwhelms the oscillator ( $\boldsymbol{F}$ is stronger than the restoring epicyclic force) causing the mode to become nearly purely growing and changing the phase relationship between components of $\boldsymbol{u}$. This produces a sudden increase in the maximum growth rate. The zero-frequency mode, where the forced-oscillator analogy in the text (Section 6.1) is most appropriate, is labelled with filled circles in each panel. The transition - i.e. the change from a mode with $\mathfrak{R}(\omega)>\Im(\omega)$ to one with $\mathfrak{R}(\omega)<\Im(\omega)$ at $\mu=1$ - is well understood using the forcedoscillator analogy, while the behaviour of the fast-growing modes with $\mu>$ 1 is explained by the model in Fig. 7.

A useful rough analogy for the lowest order out-of-phase feedback is that of a spring with a external forcing that is proportional to, but in the opposite direction to, its displacement

$$
\frac{\partial^{2}}{\partial t^{2}} x(t)+\omega_{0}^{2} x(t)=J(t), \quad J(t)=J_{0} x(t) .
$$

Here, $x(t)$ is the spring's displacement, which represents the radial velocity of the epicycle, and $J(t)$ is the external forcing, which represents the jerk (rate of change of acceleration) from the dust feedback. The frequency of the oscillator, $\omega= \pm \sqrt{\omega_{0}^{2}-J_{0}}$, decreases as the forcing becomes comparable to the restoring force, splitting into purely growing and decaying modes once $J_{0}>\omega_{0}^{2}$. This transition accounts for the step change in streaming instability properties at $\mu$ $=1$ : When the dust mass density is equal to that of the gas, its forcing on the gas becomes larger than the restoring force of the epicycle, flipping the sign of the radial gas velocity and causing a near purely growing mode. Indeed, as shown in Paper I, the frequency of the fastest-growing streaming instability modes for $\mu>1$ are simply $\omega \approx \pm i \Omega \sqrt{\mu-1}$, exactly as expected from this forced-oscillator analogy. ${ }^{6}$ In addition, the analogy predicts that the real frequency of the resonant mode at $\mu<1$ should steadily decrease with increasing $\mu$. Fig. $6-$ which shows the growth rate and real frequency of the relevant modes at $\tau_{s}=0.01$ across the $\mu=1$ transition - confirms that this is the case, as well as showing the sudden increase in $\Im(\omega)$ at $\mu=1$ (recall that at $\mu<1$, the mode is weakly growing only because of the higher-order dust interaction shown in Fig. 4, which is not included in the forced-oscillator analogy).

\subsection{Wavenumber dependence}

Our simple analogy neglects the mode's $k$ dependence, assuming that the compression of the dust by the epicycle and the force of the dust on the gas remain synced (in resonance). To understand the wavenumber dependence, we must consider, in addition to the epicycles, how the natural frequency of the dust-density advection changes as $\mu$ increases. ${ }^{7}$ Because of the feedback force on the gas $\boldsymbol{F}=\delta \rho_{d} \mathbf{w}_{s} / t_{s}$, an advected dust-density perturbation drives radial gas flows as it propagates. These, in turn, drive a compressive dust flow due to the Coriolis force, which is out of phase with the advection term $\mathbf{w}_{s} \cdot \nabla \rho_{d}$. The net effect is to slow the advection of the dust-density perturbation by a factor $(1-\mu)$. Comparing the dustmodified epicycle frequency, $\omega \approx(1-\mu)^{1 / 2} \hat{k}_{z} \Omega$ (from equation 15), to the dust-advection frequency, $\omega \approx(1-\mu) \boldsymbol{k} \cdot \mathbf{w}_{s}$, we see that the two are matched at a wavenumber that scales as $k \propto(1-\mu)^{-1 / 2}$, formally approaching infinty as $\mu \rightarrow 1$. This scaling of the fastestgrowing wavenumber is indeed seen in numerical solutions of the full dispersion relation of the $\mu<1$ streaming instability (see $\mu$ $<1$ solutions in Fig. 6). More importantly, we see that the $\mu>1$ instability occurs at very short wavelengths, where physics beyond our simple toy analogies comes into play. Indeed, as shown in Paper I, the instability's fastest-growing modes increase faster with decreasing $\tau_{s}$ than those of the low- $\mu$ instability. ${ }^{8}$

\subsection{Mode structure and growth}

A sketch of how the high- $\mu$ streaming instability operates is shown in Fig. 7. An important distinction compared to our sketches of the low$\mu$ streaming and settling instabilities (Figs 4 and 5) is that the mode in the sketch is stationary (purely growing) or slowly propagating to the right (opposite to Fig. 4). With $\mu>1$, the force from the dust perturbation generated by the azimuthal velocity (see Fig. 2) is sufficiently large to flip the sign of the radial gas velocity, aligning dust-density maxima with negative radial velocities (this is reversed from Fig. 2). This radial velocity, in turn, generates an azimuthal gas and dust velocity through the Coriolis/shear force. Because $\partial_{t} u_{y}=$ $-\Omega / 2 u_{x}+\ldots$ and the mode is dominated by its exponential growth rather than oscillations, $u_{y}$ has negative maxima near the positive $u_{x}$ maxima and vice versa. Note that the Coriolis force on the radial gas velocity $\left(\partial_{t} u_{x}=2 \Omega u_{y}+\ldots\right)$, which would tend to produce radial

\footnotetext{
${ }^{6}$ Note that the fastest-growing modes occur when $k_{z} \gg k_{x}$, so that the epicyclic frequency $-\omega_{0}$ in equation $(15)-$ is simply $\Omega$ (i.e. $\hat{k}_{z} \approx 1$ ). As shown in Paper $\mathrm{I}$, in the opposite limit $k_{x} \gg k_{z}$, the growth rate is reduced to $\hat{k}_{z} \Omega \sqrt{\mu-1}$, also exactly as expected from our simple oscillator analogy.

${ }^{7}$ Mathematically, this feedback can be worked out using perturbation theory on the dust-density eigenmode.

${ }^{8}$ Specifically, equations (A2)-(A5) of Paper I shows that the fastest growing wavenumber $k_{\max }$ behaves as $k_{\max } \sim \tau_{s}^{-5 / 4}$ to $k_{\max } \sim \tau_{s}^{-3 / 2}$, depending on the regime, as opposed to $k_{\max } \sim \tau_{s}^{-1}$ for the low- $\mu$ streaming or settling instabilities.
} 

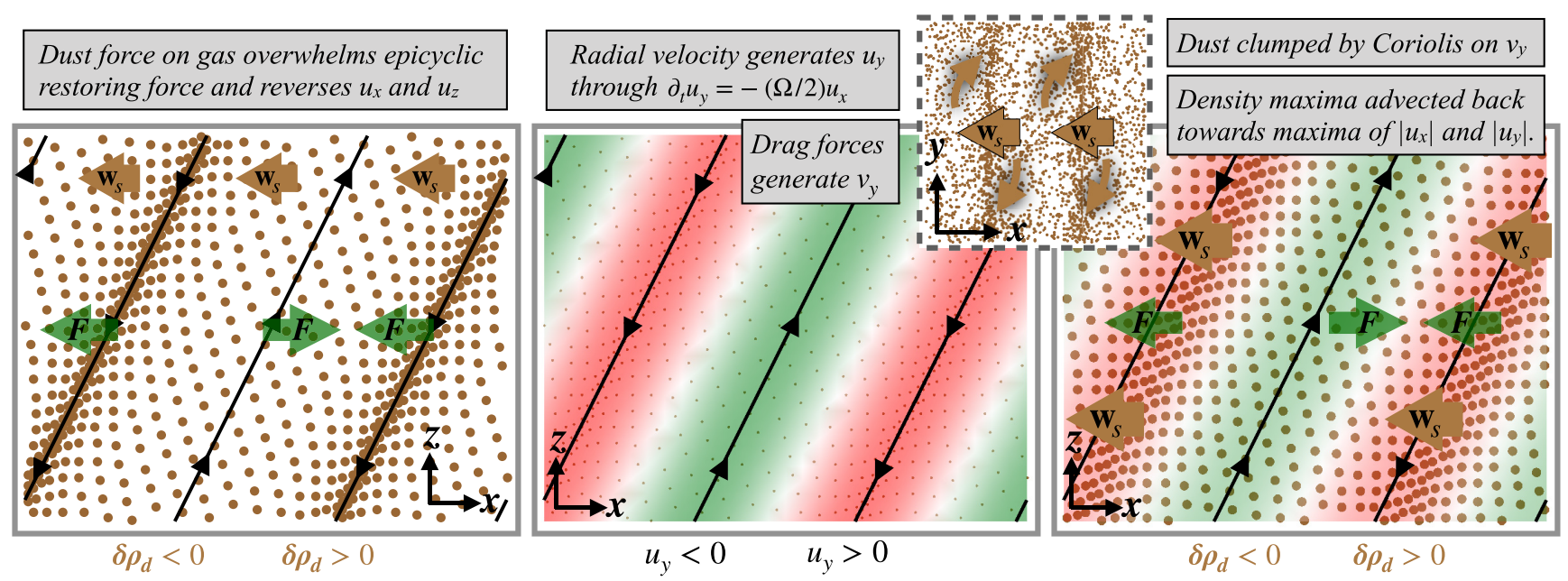

Figure 7. The high-dust-to-gas-ratio $(\mu>1)$ streaming instability. For $\mu>1$, the force of the dust-density perturbation on the gas $(\boldsymbol{F})$ overwhelms the natural restoring force of the epicycle (left-hand panel). This reverses the sign of the radial gas velocity (compare to Fig. 2), which in turn generates an azimuthal velocity through the Coriolis/shear force (middle panel). Note the different phase relationship between $u_{x}$ and $\delta \rho_{d}$, and between $u_{y}$ and $u_{x}$ compared to the unmodified epicycle shown in Fig. 2 (red and green regions show $u_{y}>0$ and $u_{y}<0$, respectively). This azimuthal velocity causes a radial compression of the dust density (see top inset and Fig. 2), which is advected inwards by the dust's drift to be back in phase with the original perturbation, thus causing instability. Note that, unlike the sketches in Fig. 4 and the left-hand panel of Fig. 5, the pictured mode is stationary (purely growing) or slowly propagating outwards (which allows the density perturbation to stay in phase with the velocity perturbations at longer wavelengths).

velocities of the opposite sign, is dominated by the dust feedback (as must be the case for the forced-oscillator analogy to be correct).

The Coriolis force on the radial dust velocity, by contrast, remains important, because this generates the necessary compressive flow to cause the dust-density perturbation that sustains the mode (top inset; see Fig. 2). In the absence of dust advection, or if the mode was propagating in sync with the density advection (as in Fig. 4), this density perturbation would be $\pi / 2$ out of phase with $u_{y}\left(\delta \rho_{d}\right.$ maxima to the right of $u_{y}$ maxima; see top inset); however, the background dust drift causes perturbations to be advected inwards at the same time as they are produced, bringing them back into alignment with the negative radial velocity and feeding back coherently on the original mode. As can be seen in Fig. 6 (blue and grey curves), at longer wavelengths, the mode propagates slowly outwards $(\Re(\omega)<0)$, against the drift, which allows the dust-density perturbation to stay in phase with $u_{x}$ by reducing the necessary inwards shift in $\delta \rho_{d}$ due to advection. At wavelengths that are shorter than that where the mode is purely growing $(\Re(\omega)>0)$, the mode is quickly killed because the density perturbation would be advected too far inwards to cause the necessary feedback on the gas. Similarly, as $\mu$ increases, the increase in mode growth rate due to the increased dust force on the gas $(\omega \propto \sqrt{\mu-1})$ requires that the mode move to shorter wavelengths because the dust-density perturbation must be advected back across the mode wavelength more rapidly (in addition, $\mathbf{w}_{s}$ decreases with $\mu$, which exacerbates the effect). This move to shorter wavelengths at increasing $\mu$ can be seen in the dispersion relation shown in Fig. 6 (compare $\mu=1.1$ and 2 solutions).

\subsection{Model summary}

Our model qualitatively explains the following important features of the streaming instability as follows:

(i) The sudden increase in the growth rate of the streaming instability at $\mu=1$, which is when the forcing on the gas from the dust overwhelms the natural restoring force of epicyclic oscillations. (ii) The maximum growth rate of the streaming instability is independent of grain size $\tau_{s}$ if $\mu>1$ (for $\tau_{s} \lesssim 1$ ).

(iii) The presence of a purely growing streaming instability if $\mu>$ 1. (Or, at somewhat longer wavelengths, the streaming instability's reversed propagation direction compared to the $\mu<1$ instability.) The highly simplified model can even predict the mode's fastest growth rate, $\omega \approx i \Omega \sqrt{\mu-1}$.

(iv) The short wavelength of the high- $\mu$ instability and the decrease of this wavelength with $\mu$. The mechanism requires that a dust-density perturbation be advected across the mode sufficiently rapidly so that the force it induces on the gas aligns with the radial gas velocity. At larger $\mu$, the faster growth rate requires that this happens more rapidly, necessitating shorter wavelengths.

(v) The high- $\mu$ streaming instability grows fastest when $k_{x} \ll k_{z}$, because the dust forcing on the gas (in the $\hat{\boldsymbol{x}}$ direction) is closely aligned with the incompressible velocity (in direction $\pm \hat{k}_{z} \hat{\boldsymbol{x}} \mp \hat{k}_{x} \hat{z}$ ). However, $k_{x} \ll k_{z}$ modes also necessitate smaller wavelengths, because short radial wavelengths are required for operation of the mode (see point iv).

(vi) Both inwards dust drift and Coriolis forces are necessary for the mechanism of the high- $\mu$ streaming instability. Dust drift exerts a force on the radial gas velocity and shifts density perturbations back in phase with the mode. Coriolis/shear forces generate azimuthal from radial velocities, and in turn, dust-density perturbations from the azimuthal dust velocity perturbations.

(vii) The gas and dust velocities in the high- $\mu$ streaming instability do not have the same structure as epicyclic oscillations. Indeed, the mode is very different in character to an epicycle, unlike the $\mu<1$ streaming instability.

Finally, the model also explains how the wavelength of the fastestgrowing low- $\mu(\mu<1)$ streaming instability mode decreases as $\mu$ increases. This occurs because the dust advection frequency decreases more rapidly with $\mu$ than the epicyclic frequency, meaning the effective resonant wavelength decreases. 


\section{CONCLUSIONS}

This article is intended to elucidate the key behaviours and origins of three related dust-drag induced instabilities: (i) the dust-gas 'streaming instability' at low dust-to-gas mass ratios $(\mu<1$; Youdin \& Goodman 2005), (ii) the disc 'settling instability' of Paper I (Squire \& Hopkins 2018b), and (iii) the streaming instability at high dust-togas-ratios $(\mu>1)$. By facilitating the coagulation of grains from the smallest dust into larger gravitationally bound objects, these instabilities are believed to play a key role in planetesimal formation in protostellar discs. Each instability derives from the combination of dust drift, rotation (Coriolis forces), and gas pressure, while the low$\mu$ streaming and settling instabilities are in the family of 'resonant drag instabilities' (RDIs; Squire \& Hopkins 2018a). However, each of the three also exhibit fundamental and qualitative differences in their physical driving mechanisms, which strongly influences their mode structure and growth rates.

Throughout the article, our philosophy has been to eschew detailed mathematical derivations in favour of representing the crucial features of the dynamical equations in the simplest way possible. Along with detailed figures that sketch the key motions and forces involved in each mode, these simplified models enable a straightforward, intuitive understanding of the instabilities' operation that has been lacking in previous literature. Further, combined with the detailed analytic derivations of growth rates and mode structure presented in Paper I, they provide a relatively complete picture of the streaming and settling instabilities' operation and how their properties change with parameters.

Our models are summarized in Fig. 1 and in Figs 4, 5, and 7, which apply, respectively, to the low- $\mu$ streaming instability, the settling instability, and the high- $\mu$ streaming instability. These figures are designed to be digestible without detailed reference to the text (see table 1 for definitions and conventions). In addition, Sections 4.1, 5.1 , and 6.4 provide a summary of the key features of each regime that are explained by our models. Some of the most important of these conclusions are as follows:

(i) Dust clumping due to the Coriolis force: Gas motions clump the dust because of the action of the Coriolis force. Azimuthal dust velocities are deflected into the radial direction by the Coriolis force, which generates a compressive radial dust flow even if the gas motions are incompressible (see Fig. 2).

(ii) Dust backreaction from density perturbations: Dust-density perturbations feed back on the gas due to the dust's mean drift. Regions of higher dust density exert a higher-than-mean force on the gas in the drift direction due to drag, while regions of lower dust density exert a lower force (see Fig. 3). This feedback allows dust clumps to re-enforce the gas motions that caused them.

(iii) Resonant modes: When gas motions dominate, at $\mu<1$, the instabilities grow fastest at the 'resonant' wavelength, where the drift speed of the dust matches the phase velocity of the wave (as formalized by RDI theory; Squire \& Hopkins 2018a). At this wavelength, a propagating gas epicycle causes strong secular growth of dust-density perturbations, because the driving of the dust matches its natural advection rate.

(iv) Strong feedback from small grains: Instabilities caused by small grains $\left(\tau_{s} \ll 1\right)$ generically grow just as rapidly as those caused by large grains ( $\tau_{s}$ approaching 1$)$. Smaller grains, although they are better coupled to the gas, also exert larger forces on the gas for the same drift speed. They thus interact more strongly with smallscale gas motions and cause fast-growing instabilities with short wavelengths. This conclusion applies to grains that are arbitrarily small, until a non-ideal effect such as viscosity starts to impact mode structure. However, the streaming instability with $\mu<1$, which is well known to have a growth rate that decreases linearly with $\tau_{s}$ at $\tau_{s}$ $<1$ (Youdin \& Goodman 2005), is unusually weak for small grains. Its low growth rate occurs because, when dust is drifting in the disc mid-plane (radially), the natural force feedback of a dust-density perturbation on a gas epicycle (point $i$ ) is nearly out of phase with the gas velocity of the epicycle. This neutralizes the leading-order backreaction term, which would otherwise drive an instability. This neutralization does not occur if the dust has non-vanishing vertical drift (the settling instability), accounting for the settling instability's larger growth rate, which is independent of grain size for smaller grains.

(v) A different streaming instability at $\mu>1$ : The streaming instability undergoes a sudden change in its properties at $\mu=$ 1 , exhibiting a much larger maximal growth rate that does not decrease with grain size (see Fig. 6). This arises because the force feedback of the dust on an epicycle, which is naturally out of phase with the epicycle's velocity (point iv), becomes so strong that it overwhelms the natural restoring force, flipping the sign of the radial gas velocity. In its simplest form, the instability does not propagate and does not resemble the mode structure of an epicycle, because it is dominated by forces from the drifting dust. The instability grows rapidly compared to the low- $\mu$ streaming instability, with a maximum growth rate $\Im(\omega) \approx \Omega \sqrt{\mu-1}$, although it operates only at very small scales for small grains.

(vi) Pressure forces: Gas pressure forces always dominate over dust backreaction forces (Section 2.2.1), implying gas motions are nearly incompressible. This further implies that a dust-density perturbation that varies only in the direction of the drift ( $\boldsymbol{k}$ parallel to $\mathbf{w}_{s}$ ) cannot drive instability, because the backreaction force from the dust is resisted by gas pressure forces (see Section 3.3.1).

Although the basic ideas of conclusions (iii)-(vi) have appeared in various forms in previous works (Paper I; Youdin \& Goodman 2005; Youdin \& Johansen 2007; Jacquet et al. 2011; Zhuravlev 2019; Jaupart \& Laibe 2020, Pan 2020), the novel feature of this work is the explanation of why these properties arise from the basic physics of epicyclic motion, gas pressure forces, and dustgas drag forces. Although it transpires that some of this physics is less straightforward than for other astrophysical fluid instabilities, the basic ideas could be useful in future work for applications including the analysis/interpretation of non-linear simulations and the development of non-linear models of streaming-instability-induced turbulence.

\section{ACKNOWLEDGEMENTS}

We would like to thank the reviewer, M. Pessah, for helpful suggestions that led to significant improvement in the manuscript. We also thank P. Benítez-Llambay, J. Goodman, and L. Krapp for helpful discussions. Support for JS was provided by Rutherford Discovery Fellowship RDF-U001804 and Marsden Fund grant UOO1727, which are managed through the Royal Society Te Apārangi. Support for PFH was provided by NSF Collaborative Research Grants 1715847and 1911233, NSF CAREER grant 1455342, and NASA grants 80NSSC18K0562 and JPL 1589742.

\section{DATA AVAILABILITY}

No new data were generated or analysed in support of this research. 


\section{REFERENCES}

Ansdell M., Williams J. P., Manara C. F., Miotello A., Facchini S., van der Marel N., Testi L., van Dishoeck E. F., 2017, AJ, 153, 240

Auffinger J., Laibe G., 2018, MNRAS, 473, 796

Bai X.-N., Stone J. M., 2010, ApJ, 722, L220

Balbus S. A., Hawley J. F., 1992, ApJ, 392, 662

Birnstiel T., Fang M., Johansen A., 2016, Space Sci. Rev., 205, 41

Carrera D., Johansen A., Davies M. B., 2015, A\&A, 579, A43

Chen K., Lin M.-K., 2020, ApJ, 891, 132

Chiang E., Youdin A. N., 2010, Ann. Rev. Planet. Earth Sci., 38, 493

Collins G. W., 1989, The Fundamentals of Stellar Astrophysics II, W. H. Freeman, New York

Goldreich P., Ward W. R., 1973, ApJ, 183, 1051

Goodman J., Pindor B., 2000, Icarus, 148, 537

Hopkins P. F., Squire J., 2018, MNRAS, 480, 2813

Hopkins P. F., Squire J., Seligman D., 2020, MNRAS, 496, 2123

Jacquet E., Balbus S., Latter H., 2011, MNRAS, 415, 3591

Jaupart E., Laibe G., 2020, MNRAS, 492, 4591

Johansen A., Oishi J. S., Mac Low M.-M., Klahr H., Henning T., Youdin A., 2007, Nature, 448, 1022

Johansen A., Youdin A., Mac Low M.-M., 2009, ApJ, 704, L75

Johansen A., Blum J., Tanaka H., Ormel C., Bizzarro M., Rickman H., 2014, Beuther H., Klessen R. S., Dullemond C. P., Henning T., eds, Protostars and Planets VI. Univ. of Arizona Press, Tucson, p. 547

Krapp L., Benítez-Llambay P., Gressel O., Pessah M. E., 2019, ApJ, 878, L30
Krapp L., Youdin A. N., Kratter K. M., Benítez-Llambay P., 2020, MNRAS, 497, 2715

Laibe G., Price D. J., 2014, MNRAS, 440, 2136

Melrose D. B., 1980, Plasma Astrohysics. Non-Thermal Processes in Diffuse Magnetized Plasmas - Vol. 1: The Emission, Absorption, and Transfer of Waves in Plasmas; Vol. 2: Astrophysical applications, Gordon and Breach, New York

Moseley E. R., Squire J., Hopkins P. F., 2019, MNRAS, 489, 325

Nakagawa Y., Sekiya M., Hayashi C., 1986, Icarus, 67, 375

Pan L., 2020, ApJ, 898, 8

Pan L., Yu C., 2020, ApJ, 898, 7

Seligman D., Hopkins P. F., Squire J., 2019, MNRAS, 485, 3991

Simon J. B., Armitage P. J., Li R., Youdin A. N., 2016, ApJ, 822, 1

Squire J., Hopkins P. F., 2018a, ApJ, 856, L15

Squire J., Hopkins P. F., 2018b, MNRAS, 477, 5011 (Paper I)

Umurhan O. M., Estrada P. R., Cuzzi J. N., ApJ, 2019, 895, 26A

Williams J. P., Cieza L. A., 2011, ARA\&A, 49, 67

Yang C. C., Johansen A., Carrera D., 2017, A\&A, 606, A80

Youdin A. N., Goodman J., 2005, ApJ, 620, 459

Youdin A. N., Johansen A., 2007, ApJ, 662, 613

Zhuravlev V. V., 2019, MNRAS, 489, 3850

Zhuravlev V. V., 2020, MNRAS, 494, 1395

This paper has been typeset from a $\mathrm{T}_{\mathrm{E}} \mathrm{X} / \mathrm{L} \mathrm{T} \mathrm{E} \mathrm{X}$ file prepared by the author. 